\title{
Binge eating, purging and non-purging compensatory behaviours decrease from adolescence to adulthood: A population-based, longitudinal study
}

Dawit Shawel Abebe ${ }^{1,2^{*}}$, Lars Lien ${ }^{3}$, Leila Torgersen ${ }^{2}$ and Tilmann von Soest ${ }^{1,2}$

\begin{abstract}
Background: Subclinical forms of eating disorders (ED) are highly prevalent, but relatively little is known about age trends, gender differences and distinctions among symptoms. This study investigates age trends and gender difference in binge eating, purging and non-purging compensatory behaviours (CB) and the relationship of such behaviours to psychosocial problems.

Methods: Data from the national representative longitudinal study "Young in Norway" (ages 14-34 years) were analysed using $\chi^{2}$ tests, logistic random intercept models and analyses of covariance.

Results: For both genders, a decrease was found in the prevalence of CB from age 14-16 years to 23 years and over. For binging, however, a significant decrease was found only for females, whose binge eating also declined more markedly over time than did males'. A significant gender difference was detected for purging, with females at higher risk. Purging was related to particularly serious symptoms of psychosocial problems: Those who purged had significantly higher levels of appearance dissatisfaction, anxiety and depressive symptoms, alcohol consumption, self-concept instability and loneliness than those with symptoms of other forms of disordered eating.

Conclusions: Individuals affected by purging need to be targeted as a high-risk group. The distinction in severity among the subclinical ED may indicate the need for the reformulation of the eating disorder not otherwise specified category in the Diagnostic and Statistical Manual of Mental Disorders-V.
\end{abstract}

\section{Background}

Binge eating and compensatory behaviours $(\mathrm{CB})$ are the most frequent symptoms of full- and sub-threshold forms of eating disorders (ED) $[1,2]$. CB are inappropriate weight control behaviours and are divided into purging behaviours such as self-induced vomiting and the use of laxatives and diuretics, and non-purging behaviours such as the use of diet pills, excessive exercise and dietary restraint. These behaviours, with or without binge eating, are the essential behavioural criteria for the current classifications of ED and represent the central features of bulimia and eating disorders not

\footnotetext{
* Correspondence: dawitabebe@nova.no

${ }^{1}$ Norwegian Social Research (NOVA), P.O. Box 3223, Elisenberg 0208 Oslo, Norway

Full list of author information is available at the end of the article
}

otherwise specified (EDNOS) [2]. However, current classification systems for ED have been criticised for assigning too large a proportion of diagnosed ED patients to the residual EDNOS category [3] - nearly half of those receiving treatment [4]. Recent studies argue that binge eating with and without purging and non-purging $C B$ needs to be more appropriately classified within diagnostic systems of ED [5-9]. Some suggest that purging without other ED symptoms (purging disorder) should be included in future classification schemes $[5,10,11]$. In contrast, others propose that future diagnostic categories be reserved for combinations of binge eating with $\mathrm{CB}$, whether purging or non-purging $[7,9]$.

In addition to clinical data, population-based studies examining the epidemiology of subclinical symptoms of binge eating and $\mathrm{CB}$ may prove informative for the

\section{Biomed Central}


future classification of ED. Although a few such studies have been published [5-9], they provide limited information concerning the prevalence of symptoms and correlates with other psychosocial variables, and also whether such symptoms have different epidemiological patterns and co-morbidity. Thus, by using data from a large population-based longitudinal cohort study in Norway, this study aims to investigate the epidemiology of binge eating and purging and non-purging $\mathrm{CB}$. It focuses particularly on age trends and gender differences in reported symptoms and the relationship of the symptoms to psychosocial problems.

Concerning age trends, few prospective communitybased studies to date have examined changes in binge eating and $\mathrm{CB}$ from adolescence to adulthood [12-14]. Heartherton and colleagues suggest that the transition to adulthood is related to a decline in binge eating and CB for women [12]. Recently, however, another study reported no significant decreases in point-of-time prevalence of purging among men and women over time, indicating stability in the frequency of purging behaviours across cohorts and time [5]. These studies did not explore age-related changes, and such exploration is vital if we are to gain an improved understanding about the progress of binge eating and CB from adolescence to young adulthood.

Unlike the full-threshold forms of ED, which have disproportionately high rates among females, binge eating and $\mathrm{CB}$ are found at comparatively high rates in both genders [15]. However, there remains a female preponderance for purging behaviours [16], whereas males and females are equally apt to report binge eating and excessive exercise [15]. Such findings from community-based studies provide ample support to capture the types of binge eating and $\mathrm{CB}$ specific to each gender.

So far, few studies have examined how specific subclinical forms of ED such as binging, binging combined with $\mathrm{CB}$, purging and non-purging are related to measures of general psychopathology. One exception is Mond and colleagues, who reported that individuals who engaged in a combination of recurrent binge eating and $\mathrm{CB}$, whether purging or non-purging, had significantly higher levels of dietary restraint, eating and weight concerns, general psychological distress and functional impairments than those engaging in $\mathrm{CB}$ alone [7]. Moreover, Haedt and Keel showed that purging behaviours per se can demonstrate distinctive psychopathology; individuals with purging behaviour reported significantly greater perfectionism and more impairment in social relationships and educational satisfaction than a non-ED group [5]. Further research on characteristics of such eating problems has been strongly recommended $[9,10]$. To validate whether binge eaters with or without $\mathrm{CB}$ are discriminable, it is important to show that these groups have significant and distinctive patterns of psychopathology.

In conclusion, more knowledge about the epidemiology of binge eating and CB is needed. This populationbased study aims to examine this issue by looking at age trends and gender differences in the prevalence of such symptoms. Moreover, it investigates how specific symptoms of binge eating and $\mathrm{CB}$ are related to a comprehensive range of psychosocial variables. These variables include measures of general eating problems and other psychosocial problems such as appearance satisfaction, anxiety and depressive symptoms, self-worth, alcohol consumption, relationship to parents, self-concept, social support and loneliness.

\section{Methods}

\section{Procedure and participants}

We analysed data from the longitudinal study Young in Norway $[17,18]$, a national representative study that was conducted at four time points: 1992 (T0), 1994 (T1), 1999 (T2) and 2005 (T3). The initial sample at T0 comprised 12287 students in grades 7-12 (12-20 years of age) from 67 representative schools in Norway. Every school in the country was included in the register from which the schools were selected, and each grade was equally represented. The sample was stratified according to geographical region and school size, which in Norway is closely related to the degree of urbanization. Each school's sampling probability was proportional to the number of students in the school, giving each student an equal probability of being selected. Detailed information about the sampling procedure is presented elsewhere $[17,18]$. The response rate at T0 was $97 \%$.

Three of the participating schools at T0 were not part of the 1994 follow-up study (T1; 14-22 years of age). At another school, a burglary in the school's archives resulted in the loss of the project's identification records. In all, then, 9679 students from 63 schools were eligible to complete the T1 questionnaire. Because a considerable proportion of the students had completed their 3-year track at the junior or senior high school they were attending at $\mathrm{T} 0$, those no longer at the same school at $\mathrm{T} 1$ received the questionnaire by mail. For this group the response rate was $68 \%$; for those at their original schools it was $92 \%$.

Only students who completed the questionnaire in school at T1 $(\mathrm{n}=3844)$ were followed up at T2 due to the comparatively lower response rate from those who received the T1 questionnaire by mail. Because the survey was originally planned as a 2-wave study, informed consent had to be obtained again at $\mathrm{T} 1$. Of the total number of consenting individuals at T1 $(\mathrm{n}=3507,91 \%)$, $2923(84 \%)$ responded to the questionnaire they received by mail at T2 (19-28 years of age). The overall 
participation rate at $\mathrm{T} 2$, based on all eligible students at T0 who still were at their original school at T1, was therefore $68 \%$.

In 2005 (T3), all those who had consented at T1 to the follow-up were again invited to participate (25-34 years of age). In all, 2890 of the $3507 \mathrm{~T} 1$ participants (83\%) completed the T3 questionnaire, resulting in an overall response rate of $67 \%$. For purposes of this study, data from T1-T3 were used, as binge eating and CB were not measured at T0.

\section{Measures}

Binging and compensatory behaviours were measured by the six items on the severity scale of the Bulimic Investigatory Test, Edinburgh (BITE) [19]. These items measure the frequency of binge eating and $\mathrm{CB}$, ranging from 'never' to '2-3 times per day.' The scale consists of the following questions: 'Do you ever binge on large amounts of food?'; 'Do you ever fast a whole day?'; 'Do you take diet pills to help you lose weight?'; 'Do you take laxatives to help you lose weight?'; 'Do you take diuretics to help you lose weight?'; 'Do you make yourself vomit to help you lose weight?'; and 'Do you train very hard to help you lose weight?'. For estimating prevalence, we set the minimum frequency of each behaviour to once per week [5] and subsequently dichotomised the frequencies into less than once per week (0) and once per week or more (1). Next, we created four groups based on patterns of binge eating and $\mathrm{CB}$. The four groups were: binging without $\mathrm{CB}$; binging combined with any form of $\mathrm{CB}$ (binging- $\mathrm{CB}$ ); purging $\mathrm{CB}$ (self-induced vomiting, taking laxatives, or taking diuretics); and non-purging $\mathrm{CB}$ (taking diet pills, fasting or exercising excessively). Individuals with binge eating symptoms were excluded from the purging $C B$ and non-purging $\mathrm{CB}$ groups, thereby ensuring that those groups consisted of individuals reporting only categoryspecific symptoms. All groups were mutually exclusive. Due to the small sample size of the purging CB group, we could not separate binge eating with $C B$ into binge eating with purging $\mathrm{CB}$ and binge eating with non-purging CB.

Eating problems were also assessed by two other general measures. The first, the BITE scale, consists of 30 items measuring a broad range of symptoms as well as attitudinal and cognitive aspects of bulimia [19]. All items were rated on a scale ranging from 1 to 4 , and a mean score was calculated, with high scores indicating high levels of symptoms. The second additional measure, the Eating Attitude Test-12 (EAT-12), measures eating problems and concerns related to dieting, bulimia and food preoccupation, and oral control [20,21]. The scale has 12 items with response alternatives ranging from 1 ('never') to 4 ('always'). Mean scores were calculated, with high scores reflecting high levels of eating problems.

Appearance satisfaction was assessed by the Body Areas Satisfaction Scale (BASS) [22]. The scale rates respondents' level of satisfaction with seven body areas: face, lower torso, mid-torso, upper torso, muscle tone, weight and height. Response options varied from 1 ('very dissatisfied') to 5 ('very satisfied'). A mean score was computed, with high scores indicating a high level of satisfaction.

Depressive symptoms were measured by the 6-item Depressive Mood Inventory constructed by Kandel and Davies [23]. Using a response scale ranging from 1 to 4 , participants were asked to restrict their ratings to the preceding week. Mean scores were calculated, with high scores indicating high levels of depressive symptoms.

Symptoms of anxiety were measured by six items derived from the Hopkins Symptom Checklist [24]. Item responses ranged from 1 to 4 and were restricted to the preceding week. A mean score was computed, with high scores showing strong symptoms of anxiety [25].

General self-worth was measured using the Global Self-Worth subscale of a revised version of the Harter's Perception Profile for Adolescents [26,27]. A 4-point response scale was applied, ranging from 1 ('corresponds very poorly') to 4 ('corresponds very well'). A mean score was computed, with high scores reflecting high self-worth.

Alcohol consumption was measured by asking participants to indicate how often they had 'drunk so much that you felt clearly intoxicated' during the preceding 12 months. The response scale ranged from 1 ('never') to 6 ('more than 50 times').

Relationship to parents was assessed by a short version of the Parental Bonding Instrument [28]. The scale measures the emotional relationship between participants and parents by focusing on two dimensions, parental care and parental overprotection. Each dimension consists of five items and has a response scale ranging from 1 ('very like') to 4 ('very unlike'). High scores on the care subscale indicate a parent-child relationship based on emotional warmth, closeness and empathy, whereas high scores on the overprotection subscale suggest parental obstruction of independent behaviour, parental control and parental intrusion [28].

Self-concept was measured by a revised version of Rosenberg's Stability of Self Scale [29]. The scale has four items, each with a response range from 1 to 4 . Low mean scores indicate stability and high scores instability in terms of self-concept $[29,30]$.

Social support was measured by five items of the Social Support Questionnaire, modeled after Sarason and colleagues' scale [31]. The response alternatives range from 1 ('very poorly satisfied') to 4 ('very 
satisfied'). High mean scores indicate respondents' high level of satisfaction with their social support network.

Loneliness was measured by a 5 -item version of the UCLA Loneliness Scale, each item having response options ranging from 1 ('never') to 4 ('often') [32]. A higher mean score reflects greater loneliness.

Body mass index (BMI, $\mathrm{kg} / \mathrm{m} 2$ ) was computed from self-reported measures of height and weight. Selfreported BMI has been demonstrated to be a valid measure of actual BMI [33]. Age was recorded at the time of each survey. Gender was coded as 1 for male and 2 for female.

\section{Statistical analysis}

We applied $\chi^{2}$ tests to determine the significance of the differences in prevalence of binge eating and $\mathrm{CB}$ at each study time and between males and females. We also carried out $\chi^{2}$ tests for trends to assess changes in odd ratios (OR) over time and age. A logistic random intercept model was applied to investigate age- and gender-related changes in binge eating and $\mathrm{CB}$, and analyses of covariance (ANCOVA) were performed to compare groups on continuous measures of general eating and psychosocial problems, controlling for age and gender as covariates. The Tukey-Kramer test was applied for multiple post hoc comparisons of groups. Statistical analysis of the data was carried out using Stata SE/11 for Windows.

\section{Attrition analysis}

Since a large proportion of the sample did not respond to the questionnaires at T2 and T3, analyses were conducted to explore the potential impact of certain variables on attrition. Specifically, we performed univariate logistic regression to investigate whether binge eating and/or $\mathrm{CB}$ at $\mathrm{T} 1$ predicted drop out at $\mathrm{T} 2$ or $\mathrm{T} 3$. Results of these analyses showed, with one exception, no significant differences in proportions of binge eating and all types of CB between those who dropped out and those who stayed in the study $(p>0.05)$. The exception was that participants who exercised excessively at T1 had a lower risk of drop out at T2 and T3 $(\mathrm{OR}=0.76$, $95 \% \mathrm{CI}=0.66-0.87, p<0.01$ at $\mathrm{T} 2$ and $\mathrm{OR}=0.75,95 \%$ $\mathrm{CI}=0.65-0.85, p<0.01$ at $\mathrm{T} 3)$.

\section{Ethical clearance}

The study was approved by the Norwegian Data Inspectorate and the Regional Committee for Medical Health Research Ethics. Principles governing biomedical research in humans as stated in the Declaration of Helsinki were followed.

\section{Results}

The mean ages of participants were 17.2, 21.9 and 28.3 years at T1, T2 and T3, respectively. Gender-specific prevalence for binge eating and all forms of CB across all three time points are summarized in Table 1 . We found statistically significant trends for binge eating and fasting among males; the prevalence of these behaviours decreased from T1 to T3. For females, the prevalence of binge eating, excessive exercise and fasting decreased significantly from T1 to T3. For both genders, however, the use of diet pills increased significantly over time. Females reported a significantly higher prevalence than males of binge eating and self-induced vomiting at $\mathrm{T} 1$, use of diet pills at T2 and T3 and excessive exercise at $\mathrm{T} 1$ and T3. In contrast, we found no significant gender difference in the taking of laxatives and diuretics and fasting at each study time. Nonetheless, it is important to note that some of groups have a small sample (n).

Figures 1 and 2 illustrate changes for males and females, respectively, in the prevalence of binge eating and $\mathrm{CB}$ for our four subclinical ED subgroups from adolescence to young adulthood. To ensure an adequate sample size in each age group and to describe changes in prevalence at specific ages, we categorised the age of participants into 14-16, 17-19, 20-22, and 23 years and above. Chi square tests showed significant trends for the purging and non-purging $\mathrm{CB}$ groups for both females (purging: $\chi^{2}(1)=5.8, \mathrm{p}=0.016$; non-purging: $\left.\chi^{2}(1)=55.2, p<0.001\right)$ and males (purging: $\chi^{2}(1)=$ 4.2, $p=0.040$; non-purging: $\left.\chi^{2}(1)=101.8, p<0.001\right)$, indicating a decrease in the prevalence of these behaviours from age 14-16 to age 23 and over for both genders. For the binging without $\mathrm{CB}$ and binging- $\mathrm{CB}$ groups, we found significant decreases in prevalence from age 14-16 to age 23 and over for females (binging without CB: $\chi^{2}(1)=34.9, p<0.001$; binging-CB: $\chi^{2}(1)$ $=12.4, p<0.001)$ but no significant changes for males $(p>0.05)$.

To further address the effects of age and gender, logistic random intercept models were estimated, with age and gender as independent variables. Table 2 presents univariate regression estimates of age and gender effects for each of the four groups. Due to a high collinearity between age and time, we did not control for the time of measurement effects. These analyses controlled for dependency between repeated observations. To run such longitudinal models, we limited the analyses to participants who had responded to at least two of the three questionnaires from T1 to T3 $(\mathrm{n}=3053)$. The univariate models show that age had a significant effect on each of the four symptom variables; risks of binge eating and $\mathrm{CB}$ declined with age. A significant gender difference was found only for purging, with females at higher risk. We also tested the effect of gender $x$ age interactions in multiple regression models and found that the interaction term was significant only for the group displaying symptoms of binging without $\mathrm{CB}(\mathrm{OR}=0.90,95 \% \mathrm{CI}=$ 
Table 1 Prevalence of binge eating and compensatory behaviours by time points and gender1

\begin{tabular}{|c|c|c|c|c|c|c|c|}
\hline & \multicolumn{2}{|c|}{ T1 } & \multicolumn{2}{|c|}{$\mathrm{T} 2$} & \multicolumn{2}{|c|}{ T3 } & \multirow[t]{2}{*}{$\chi^{2}$ for time trend (d.f. 1 ) } \\
\hline & $\mathrm{N}$ & $\%$ & $\mathrm{n}$ & $\%$ & $\mathrm{n}$ & $\%$ & \\
\hline Males & 3771 & 46.9 & 1207 & 44 & 1194 & 43.8 & - \\
\hline Binge eating ${ }^{a}$ & 368 & 13.8 & 79 & 7.9 & 56 & 6.1 & $49.9^{* * *}$ \\
\hline \multicolumn{8}{|l|}{ CB - purging: } \\
\hline Self-induced vomiting ${ }^{a}$ & 8 & 0.2 & 0 & 0.0 & 2 & 0.2 & $0.5^{\mathrm{ns}}$ \\
\hline Taking laxatives & 9 & 0.2 & 1 & 0.1 & 0 & 0 & $3.7^{\mathrm{ns}}$ \\
\hline Taking diuretics & 8 & 0.2 & 2 & 0.2 & 4 & 0.3 & $0.4^{\mathrm{ns}}$ \\
\hline \multicolumn{8}{|l|}{ CB - non-purging: } \\
\hline Taking diet pills ${ }^{b, c}$ & 12 & 0.3 & 3 & 0.2 & 10 & 0.8 & $4.6^{*}$ \\
\hline Excessive exercise $^{a, c}$ & 430 & 11.7 & 113 & 9.5 & 162 & 13.6 & $1.4^{\mathrm{ns}}$ \\
\hline Fasting & 35 & 1.3 & 4 & 0.4 & 5 & 0.5 & $6.3^{* *}$ \\
\hline Females & 4273 & 53.1 & 1536 & 56 & 1530 & 56.2 & - \\
\hline Binge eating & 491 & 16.8 & 60 & 6.9 & 50 & 5.6 & $95.7^{* * *}$ \\
\hline \multicolumn{8}{|l|}{ CB - purging: } \\
\hline Self-induced vomiting & 55 & 1.3 & 27 & 1.8 & 10 & 0.6 & $2.3^{\mathrm{ns}}$ \\
\hline Taking laxatives & 9 & 0.2 & 7 & 0.5 & 4 & 0.3 & $0.3^{\text {ns }}$ \\
\hline Taking diuretics & 12 & 0.3 & 6 & 0.4 & 3 & 0.2 & $0.1^{\mathrm{ns}}$ \\
\hline \multicolumn{8}{|l|}{ CB - non-purging: } \\
\hline Taking diet pills & 20 & 0.5 & 23 & 1.5 & 31 & 2.1 & $30.1^{* * *}$ \\
\hline Excessive training & 602 & 14.3 & 178 & 11.7 & 143 & 9.4 & $25.9^{* * *}$ \\
\hline Fasting & 25 & 0.8 & 2 & 0.2 & 1 & 0.1 & $8.1^{* *}$ \\
\hline
\end{tabular}

${ }^{*} p<.05,{ }^{* *} p<.01,{ }^{* * *} p<.001, n$ number, ns non significant, $d f$ degrees of freedom, CB Compensatory behaviours, $T 1, T 2$, and $T 3$ time points 1,2 and 3 , respectively

${ }^{1}$ Prevalence is assigned only to those individuals who had behaviours at least once per week.

${ }^{\text {a }}$ Significant gender differences at T1 $(p<.05)$

${ }^{\mathrm{b}}$ Significant gender differences at T2 $(p<.05)$

'Significant gender differences at T3 $(p<.05)$

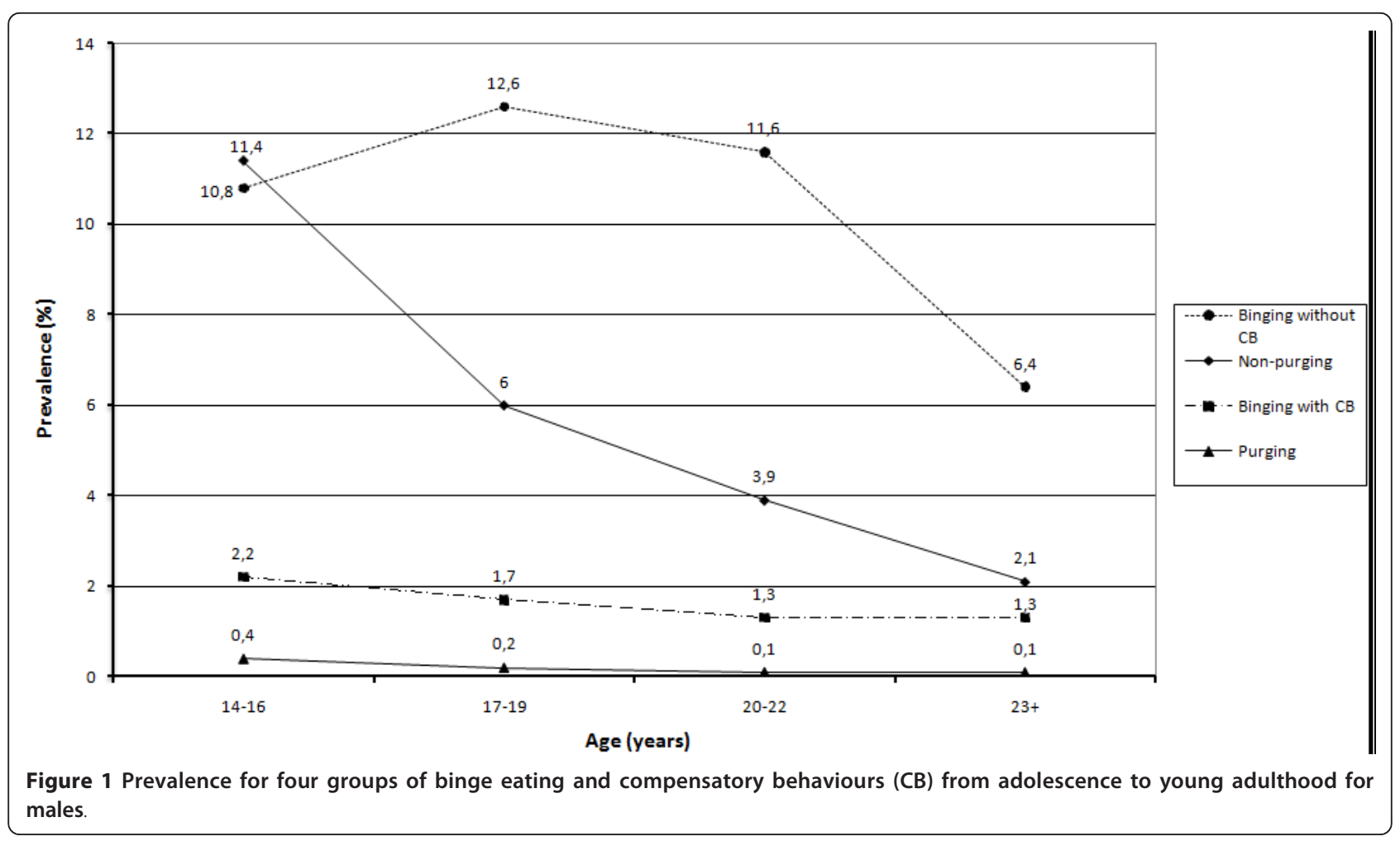




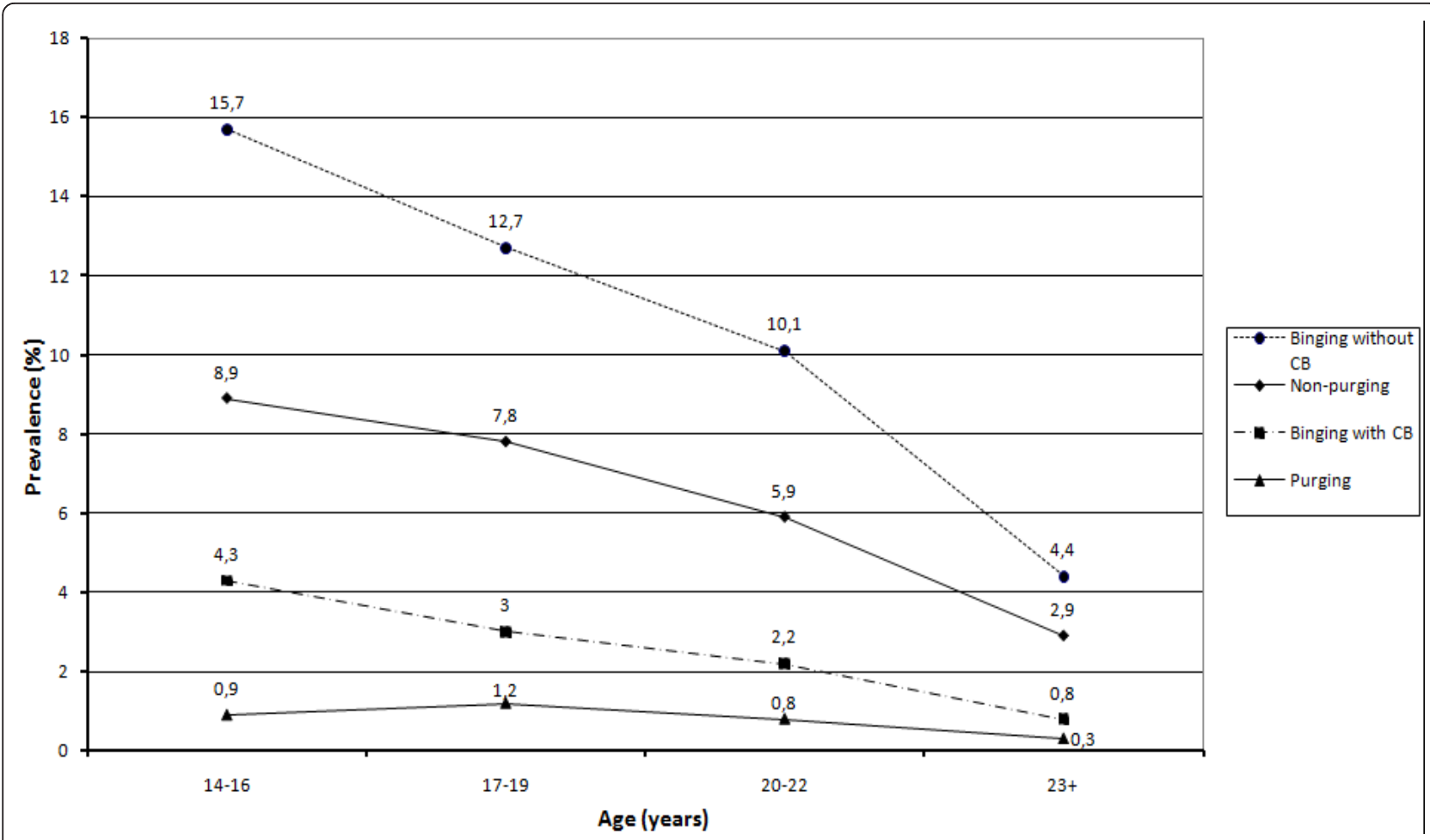

Figure 2 Prevalence for four groups of binge eating and compensatory behaviours (CB) from adolescence to young adulthood for females.

0.83-0.97, $p=0.009$ ); binge eating declined more markedly for females than for males.

We then conducted further analyses (ANCOVA) to examine whether the four groups differed on measures of general eating and psychosocial problems, using age and gender as covariates. In addition, we compared the findings from these analyses with corresponding findings from participants who reported no symptoms of either binge eating or $\mathrm{CB}(\mathrm{NoS})$. Due to the comparatively small sample sizes in the purging and binging-CB groups at T2 and T3, the analyses were based on data from T1 only.

As Table 3 shows, all scores for measures of general eating and psychosocial problems, with the exception of overprotection and social support, differed significantly among the groups. The post hoc values shown in Table 4 indicate that the purging $\mathrm{CB}$ group had significantly lower levels of appearance satisfaction and parental care, more anxiety and depressive symptoms and higher scores on the EAT-12 and BITE-30 scales than all other groups. Purgers also reported more alcohol consumption than the binging without $\mathrm{CB}$ group and those with no symptoms for binging or $\mathrm{CB}$, and higher scores on instability of self-concept and loneliness when compared with the binging without $\mathrm{CB}$, non-purging and no-symptom groups. The binging- $\mathrm{CB}$ and non-purging $\mathrm{CB}$ groups had comparable scores on most of general eating and psychosocial measures; we found no significant differences between these two groups on any of the variables. Both groups reported significantly lower levels of appearance satisfaction, more anxiety and depressive symptoms, higher scores on the EAT-12 and BITE-30 scales and a higher score for instability of self-concept than the binging without $\mathrm{CB}$ and no-symptom groups. In contrast, when compared to the other three groups, the binging without CB group showed a significantly

Table 2 Logistic random intercept model results for age and gender effects on binge eating and compensatory behaviours groups

\begin{tabular}{lcccc}
\hline Variables & Binging without CB & Binging with CB & Purging & Non-purging \\
\cline { 2 - 5 } & OR $(\mathbf{9 5} \% \mathrm{Cl})$ & OR $(\mathbf{9 5} \% \mathrm{CI})$ & OR $\mathbf{9 5 \%} \mathrm{Cl})$ & OR $(\mathbf{9 5} \% \mathrm{Cl})$ \\
\hline Age & $0.89(0.86-0.93)^{* * *}$ & $0.91(0.85-0.98)^{*}$ & $0.91(0.84-0.98)^{*}$ & $0.86(0.84-0.89)^{* * *}$ \\
Gender & $1.01(0.73-1.41)$ & $1.24(0.69-2.21)$ & $3.51(1.35-9.11)^{*}$ & $1.17(0.88-1.55)$ \\
\hline
\end{tabular}

${ }^{*} p<.05,{ }^{* *} p<.01,{ }^{* * *} p<.001 ; O R$ odds ratio, $C$ confidence interval, $C B$ compensatory behaviours 
Table 3 ANCOVA results for rating groups of binge eating and compensatory behaviours on measures of general eating and psychosocial problems 1

\begin{tabular}{|c|c|c|c|c|c|c|}
\hline \multirow[t]{2}{*}{ Variables } & $\begin{array}{l}\text { Binge eating without CB } \\
(n=726)\end{array}$ & $\begin{array}{c}\text { Binge eating with CB } \\
(\mathrm{n}=133)\end{array}$ & $\begin{array}{c}\text { Purging CB } \\
(\mathrm{n}=56)\end{array}$ & $\begin{array}{c}\begin{array}{c}\text { Non-Purging } C B \\
(n=624)\end{array} \\
\end{array}$ & $\begin{array}{c}\text { NoS } \\
(n=4016) \\
\end{array}$ & ANCOVA \\
\hline & Mean (s.d.) & Mean (s.d.) & Mean (s.d.) & Mean (s.d.) & Mean (s.d.) & $F$ (d.f. 4) \\
\hline $\mathrm{BMI}$ & $21.40(4.80)$ & $21.78(2.83)$ & $21.86(3.04)$ & $22.03(2.77)$ & $21.38(4.81)$ & $5.26^{* *}$ \\
\hline Appearance satisfaction & $3.47(0.64)$ & $3.29(0.75)$ & $2.82(0.88)$ & $3.37(0.69)$ & $3.52(0.64)$ & $16.98^{* * *}$ \\
\hline Anxiety symptoms & $1.46(0.49)$ & $1.64(0.58)$ & $1.98(0.73)$ & $1.53(0.51)$ & $1.45(0.46)$ & $14.29^{* * *}$ \\
\hline Depressive symptoms & $1.78(0.59)$ & $2.02(0.74)$ & $2.36(0.74)$ & $1.85(0.64)$ & $1.74(0.59)$ & $16.75^{* * *}$ \\
\hline Self-worth & $2.56(0.33)$ & $2.48(0.38)$ & $2.54(0.47)$ & $2.54(0.34)$ & $2.57(0.32)$ & $2.52^{*}$ \\
\hline Alcohol consumption & $2.67(1.64)$ & $2.77(1.63)$ & $3.40(1.58)$ & $2.86(1.63)$ & $2.79(1.66)$ & $5.60^{* *}$ \\
\hline EAT-12 & $1.55(0.35)$ & $1.88(0.44)$ & $2.43(0.63)$ & $1.85(0.41)$ & $1.54(0.36)$ & $184.67^{* * *}$ \\
\hline BITE-30 & 1.49(0.39) & $1.65(0.52)$ & $2.17(0.59)$ & $1.71(0.43)$ & $1.50(0.41)$ & $61.79^{* * *}$ \\
\hline \multicolumn{7}{|l|}{ Relationship to parents: } \\
\hline Overprotection & $2.03(0.58)$ & $2.01(0.60)$ & $2.09(0.69)$ & $2.04(0.59)$ & $2.01(0.57)$ & $0.41^{\mathrm{ns}}$ \\
\hline Care & $3.09(0.56)$ & $3.07(0.58)$ & $2.72(0.67)$ & $3.07(0.58)$ & $3.08(0.57)$ & $5.69^{* *}$ \\
\hline Instability of self-concept & $2.48(0.73)$ & $2.69(0.74)$ & $2.91(0.70)$ & $2.65(0.70)$ & $2.43(0.72)$ & $16.06^{* * *}$ \\
\hline Social support & $3.48(0.46)$ & $3.49(0.51)$ & $3.41(0.52)$ & $3.47(0.48)$ & $3.46(0.46)$ & $0.56^{\mathrm{ns}}$ \\
\hline Loneliness & $1.81(0.56)$ & $1.91(0.58)$ & $2.17(0.69)$ & $1.84(0.56)$ & $1.83(0.56)$ & $4.52^{*}$ \\
\hline Age (not controlled for covariates) & $17.12(1.93)$ & $16.91(1.91)$ & $17.26(2.06)$ & $16.92(2.01)$ & $17.16(1.92)$ & $2.63^{*}$ \\
\hline
\end{tabular}

${ }^{*} p<.05,{ }^{* *} p<.01,{ }^{* * *} p<.001$; ns non-significant, SD standard deviation, $d f$ degree of freedom, BMI body mass index, EAT-12 Eating Attitude Test, BITE Bulimic Investigatory Test, Edinburgh; BMI body mass index, EAT-12 Eating Attitude Test, $C B$ compensatory behaviours; NoS - individuals who did not report either binge eating or compensatory behaviours

ANCOVA - Analysis of Covariance with age and gender as covariates

${ }^{1}$ Results are based on data from the first time point only (T1: 14-22 years of age)

Table 4 Tukey-Kramer post hoc comparisons of the groups of binge eating and compensatory behaviours1

\begin{tabular}{|c|c|c|c|c|c|c|c|c|c|c|}
\hline Variables & $\begin{array}{c}\text { BEO v. BE- } \\
\text { CB }\end{array}$ & $\begin{array}{c}\text { BEO v. P- } \\
\text { CB }\end{array}$ & $\begin{array}{l}\text { BEO vs. } \\
\text { NP-CB }\end{array}$ & $\begin{array}{l}\text { BEO v. } \\
\text { NoS }\end{array}$ & $\begin{array}{l}\text { BE-CB v. } \\
\text { P-CB }\end{array}$ & $\begin{array}{c}\text { BE-CB v. } \\
\text { NP-CB }\end{array}$ & $\begin{array}{c}\text { BE-CB v. } \\
\text { NoS }\end{array}$ & $\begin{array}{l}\text { P-CB v. } \\
\text { NP-CB }\end{array}$ & $\begin{array}{l}\text { P-CB v. } \\
\text { NoS }\end{array}$ & $\begin{array}{c}\text { NP-CB v. } \\
\text { NoS }\end{array}$ \\
\hline$\overline{\mathrm{BMI}}$ & 1.81 & 0.90 & $5.67^{* *}$ & 2.48 & 0.33 & 1.41 & 0.80 & 1.39 & 0.15 & $4.96^{* *}$ \\
\hline $\begin{array}{l}\text { Appearance } \\
\text { satisfaction }\end{array}$ & $4.68^{* *}$ & $11.85^{* *}$ & $5.57^{* *}$ & 1.54 & $7.31^{* *}$ & 1.54 & $5.73^{* *}$ & $9.59^{* *}$ & $12.75^{* *}$ & $8.77^{* *}$ \\
\hline Anxiety symptoms & $5.48^{* *}$ & $12.15^{* *}$ & $4.02^{*}$ & 0.53 & $7.18^{* *}$ & 3.20 & $6.12^{* *}$ & $10.51^{* *}$ & $12.75^{* *}$ & $5.76^{* *}$ \\
\hline $\begin{array}{l}\text { Depressive } \\
\text { symptoms }\end{array}$ & $6.65^{* *}$ & $12.19^{* *}$ & $5.26^{* *}$ & 0.40 & $6.40^{* *}$ & 3.67 & $6.92^{* *}$ & $10.06^{* *}$ & $12.77^{* *}$ & $6.41^{* *}$ \\
\hline Self-worth & $4.26^{*}$ & 1.25 & 2.29 & 0.73 & 1.56 & 2.95 & $4.91^{* *}$ & 0.31 & 1.53 & 3.72 \\
\hline $\begin{array}{l}\text { Alcohol } \\
\text { consumption }\end{array}$ & 1.63 & $5.27^{* *}$ & $4.89^{* *}$ & $4.87^{* *}$ & 3.52 & 1.11 & 0.46 & 3.29 & $3.93^{*}$ & 1.59 \\
\hline EAT-12 & $16.07^{* *}$ & $28.53^{* *}$ & $25.33^{* *}$ & 0.39 & $14.65^{* *}$ & 1.79 & $17.07^{* *}$ & $18.29^{* *}$ & $29.41^{* *}$ & $32.66^{* *}$ \\
\hline BITE-30 & $6.26^{* *}$ & $19.94^{* *}$ & $17.14^{* *}$ & 3.07 & $13.31^{* *}$ & 3.35 & $5.32^{* *}$ & $13.06^{* *}$ & $19.67^{* *}$ & $19.31^{* *}$ \\
\hline \multicolumn{11}{|c|}{ Relationship to parents: } \\
\hline Overprotection & 0.93 & 1.73 & 1.22 & 0.08 & 2.05 & 1.57 & 1.02 & 1.24 & 1.76 & 1.48 \\
\hline Care & 0.16 & $6.03^{* *}$ & 0.22 & 0.37 & $5.23^{* *}$ & 0.28 & 0.32 & $5.89^{* *}$ & $6.11^{* *}$ & 0.05 \\
\hline $\begin{array}{l}\text { Instability of self- } \\
\text { concept }\end{array}$ & $4.49^{*}$ & $7.20^{* *}$ & $6.36^{* *}$ & 2.87 & 3.40 & 0.97 & $5.96^{* *}$ & $4.65^{* *}$ & $8.25^{* *}$ & $10.70^{* *}$ \\
\hline Social support & 0.99 & 0.44 & 0.88 & 0.62 & 0.99 & 0.51 & 1.32 & 0.78 & 0.27 & 1.72 \\
\hline Loneliness & 2.43 & $5.41^{* *}$ & 1.68 & 2.16 & 3.12 & 1.47 & 1.68 & $4.71^{* *}$ & $4.95^{* *}$ & 0.19 \\
\hline
\end{tabular}

*Significant differences among groups at $p<.05$

**Significant differences between groups at $p<.01$

${ }^{1}$ Tukey-Kramer test values were derived from the analysis of covariance in Table 3

$B M I$ body mass index, EAT-12 Eating Attitude Test, BITE Bulimic Investigatory Test, Edinburgh, $B E O$ binge eating only (without $C B$ ), $B E-C B$ binge eating combined with any of compensatory behaviour, $P-C B$ purging behaviours, NP-CB non-purging behaviours; NoS - participants who did not report any binge eating and or behaviours 
higher level of appearance satisfaction, and lower scores for anxiety and depressive symptoms, instability of selfconcept and the EAT-12 and BITE-30 scales. Furthermore, individuals in the binging without $\mathrm{CB}$ group did not differ significantly from participants without ED symptoms on any of the variables except alcohol consumption, on which they scored significantly higher.

\section{Discussion}

The main findings in this population-based longitudinal study are that the subclinical groups of individuals with different combinations of ED symptoms displayed distinct age- and gender-related trends. Females showed a significantly higher risk than males for purging behaviours. The prevalence of binge eating and $C B$ in females and $\mathrm{CB}$ in males gradually declined with the transition from adolescence to young adulthood. Furthermore, individuals with subclinical forms of ED differed significantly on measures of general eating and psychosocial problems. Specifically, in terms of our psychosocial measures, purging emerged as the most serious type of behaviour when compared with binging without $\mathrm{CB}$, binging- $\mathrm{CB}$ and non-purging $\mathrm{CB}$. On the other hand, individuals in the binging without $\mathrm{CB}$ group were the least disturbed and in most aspects comparable to the reference group, i.e. those without inappropriate eating behaviours.

To our knowledge, this is the first population-based study documenting the relationship between distinct types of ED behaviour (binging without $\mathrm{CB}$, binging- $\mathrm{CB}$, purging and non-purging) and different measures of general eating and psychosocial problems. The study shows a particularly high symptom load in individuals who purged, since those who purged, largely by selfinduced vomiting, had significantly higher levels of appearance dissatisfaction, anxiety and depressive symptoms, alcohol consumption, self-concept instability and loneliness. A national study of high school students in the USA also reported vomiting for weight control as the most clinically significant behaviour and one that may be a particularly deleterious component of ED [34]. In this respect, individuals engaging in purging behaviours should probably be targeted as a high-risk group.

In contrast, Mond and colleagues reported that the combination of routine binge eating and CB was associated with higher ED psychopathology and impairment in mental and physical functioning than was the occurrence of $\mathrm{CB}$ alone, regardless of the type, i.e. purging or non-purging [8]. These divergent findings may be the result of different definitions for binge eating and different scales for measuring psychopathology. We defined binge eating only in terms of the amount of food consumed. Mond et al., on the other hand, integrated loss of control in their definition, and this could add an important dimension to the relationship between binge eating and psychological disturbance. As well, Mond et al. used the Eating Disorder Examination Questionnaire and the Short-Form Disability Scale, whereas we used a wider range of eating and psychosocial measurements, providing in-depth information about the differences among the groups. In general, both community- and clinical-based studies indicate that individuals with purging subtype of bulimia nervosa have more severe psychopathology than those with non-purging bulimia nervosa and binge eating disorder, thereby demonstrating that purging behaviors may increase the severity level of psychopathology among those with the fullthreshold ED $[35,36]$. These studies support our finding that purging behaviors are related to particularly serious levels of psychosocial problems. Due to few participants with a combination of binging and purging in our study, we could not investigate how individuals with purging only symptoms differed from those with a combination of purging and binging. More research would help to clarify distinctions between these groups, which, in turn, could have important implications for the future classification of subclinical ED.

To an extent, findings from this study parallel those of earlier community-based studies [16,37]. The prevalence of binge eating and several CB behaviours appears to be higher among females than males. The gender difference in the prevalence of symptoms was especially notable for purging, with females at a significantly higher risk. A possible explanation is that females have a stronger desire to lose weight and a higher drive for thinness than males, leading them to engage in more purging behaviours [16,37]. Alternatively, males may be more uncomfortable reporting purging behaviours than females [38].

In both genders, the age-related decline in prevalence rates for binge eating and CB may be partially explainable by roles associated with the transition to adulthood, specifically partnering and motherhood (for females) $[17,39]$. However, it may be possible that psychological problems which in many cases are associated with disordered eating may not be reduced to a comparable degree as disordered eating symptoms in the transition to adulthood. For instance, even though Patton et al. reported most part of adolescent eating disorders to be limited to the teens, co-morbid conditions, such as depression, anxiety and binge drinking persisted to a much larger degree into adulthood [14]. Such findings may indicate that the decline of symptoms of disordered eating not necessarily is related to symptom alleviation for other mental health problems.

Moreover, even though we saw this declining trend for both males and females, binge eating among females stands out in terms of the magnitude of the reduction 
over age. This may be related to a greater perception on the part of females that binge eating is a problematic behaviour [40]. Along the same line of thought, males report a more positive affect (feeling happy) than females after binging [41,42].

Overall, the prevalence rates of symptoms in this study are lower when compared with the rates from two studies of US college men and women $[43,44]$. They are, however, similar to rates found in two community-based studies conducted in Australia and the USA, respectively $[5,8]$. Nonetheless, factors such as the use of different assessment measures, lack of standard definitions for syndromes, differences in methodological approaches and characteristics of sample populations may limit the comparability of the findings.

This study has a number of limitations that warrant consideration. First, the BITE does not specify a time frame for the occurrence of symptoms, thereby providing somewhat limited information about the duration of a respondent's ED. Second, binge eating determined solely by the amount of food consumed may be a somewhat limited indicator for binge eating psychopathology. The inclusion of additional BITE items such as feeling distress after binging, binging alone, and urge to binge or loss of control might better distinguish binging from normal eating. This matter bears further investigation. Third, even though it has been argued that full-syndrome ED may not differ qualitatively from sub-threshold levels $[45,46]$, it remains to be seen whether the findings from this study will be supported in studies using diagnostic categories of ED and in those using diagnostic interviews. Fourth, age-related differences in prevalence rates of ED behaviours may reflect time of measurement effects and differences in exposure to risk factors rather than effects associated with growing into adulthood. Other studies suggest that a wide range of ED behaviours remain relatively stable over time, supporting the negligible effect of time on the trend of prevalence $[5,47]$. Furthermore, separating the effect of age from period and cohort through statistical model estimation has proven problematic and has led to incorrect conclusions [48]. Fifth, limited statistical power resulting from the small sample sizes should be considered. More specifically, because the number of participants in the purging $\mathrm{CB}$ and binging$\mathrm{CB}$ groups was rather small, potential differences among the groups might not have been detected. Sixth, to obtain more differentiated information, the binging-CB group should ideally have been divided into those who binged and purged and those who binged and engaged in nonpurging CB. Unfortunately, the small number of participants reporting both binging and purging behaviours precluded such analyses. Finally, we only followed about $25 \%$ of the representative sample at T0. Even though most of the attrition was planned, and that attrition analyses showed no significant differences between those who dropped out and those who completed the study, the large proportion of drop out at the follow-up could be a source of bias.

\section{Conclusion}

Our findings suggest that purging behaviours are the most severe and gender-bound subclinical forms of ED, i.e. behaviours indicating high-risk individuals. A substantial proportion of adolescents report binge eating and inappropriate weight control methods; the risk declines to some extent with the transition to adulthood. Early preventive interventions of such inappropriate eating behaviours have the potential to decrease the likelihood of progression to full-threshold ED. The differences found among the groups in this study may be useful in the discussion concerning a reformulation of clinical forms of ED in the Diagnostic and Statistical Manual of Mental Disorders-V, particularly around the necessity to capture diversities in the EDNOS category. It is important to explore more closely whether psychosocial disturbances cause or mediate the observed difference among the ED groups. Moreover, future studies should be aimed at finding factors that are potent in distinguishing individuals who experience disordered eating symptoms which are limited to the adolescent years from those who suffer from longer lasting symptoms which persist into adulthood. Research is as well needed examining in which way psychosocial correlates of disordered eating that were found in the present study are causally related to different types of eating disordered symptoms. Such studies would provide valuable information for prevention and intervention efforts in the field of disordered eating.

\section{Acknowledgements}

This research was funded by the Norwegian Research Council (grant \# 196226 V50). The authors acknowledge and thank the staffs of Youth section at the Norwegian Social Research (NOVA) and Gertrud Hafstad (Post-doc), Norwegian Institute of Public Health for their continuous support and comments on the draft.

\section{Author details}

${ }^{1}$ Norwegian Social Research (NOVA), P.O. Box 3223, Elisenberg 0208 Oslo, Norway. ${ }^{2}$ Division of Mental Health, Norwegian Institute of Public Health, Oslo, Norway. ${ }^{3}$ Division of Mental Health and Addiction, University of Oslo, Oslo, Norway.

\section{Authors' contributions}

DSA analysed the data, interpreted the results and drafted the manuscript. All authors participated in the write-up, contributed to the interpretation of the study results and approved the final version of the manuscript submitted for publication.

\section{Competing interests}

The authors declare that they have no competing interests. 


\section{References}

1. Ackard DM, Fulkerson JA, Neumark-Sztainer D: Prevalence and utility of DSM-IV eating disorder diagnostic criteria among youth. Int J Eat Disord 2007, 40:409-417.

2. Association AP: Diagnostic and Statistical Manual of Mental Disorders Washington, DC: American Psychiatric Association; 2000.

3. Wilfley DE, Bishop ME, Wilson GT, Agras WS: Classification of eating disorders: Toward DSM-V. Int J Eat Disord 2007, 40:S123-S129.

4. Fairburn CG, Bohn K: Eating disorder NOS (EDNOS): an example of the troublesome "not otherwise specified" (NOS) category in DSM-IV. Behav Res Ther 2005, 43:691-701.

5. Haedt AA, Keel PK: Comparing definitions of purging disorder on point prevalence and associations with external validators. Int J Eat Disord 2010, 43:433-439.

6. Keel PK, Haedt A, Edler C: Purging disorder: An ominous variant of bulimia nervosa? Int J Eat Disord 2005, 38:191-199.

7. Mond J, Hay P, Rodgers B, Owen C, Crosby R, Mitchell J: Use of extreme weight control behaviors with and without binge eating in a community sample: implications for the classification of bulimic-type eating disorders. Int J Eat Disord 2006, 39:294-302.

8. Mond JJ, Hay PJ, Rodgers B, Owen C, Mitchell J: Correlates of the use of purging and non-purging methods of weight control in a community sample of women. Aust N Z J Psychiatry 2006, 40:136-142.

9. Mond JM, Hay PJ: Use of extreme weight-control behaviors in the absence of binge eating with and without subjective bulimic episodes: A community-based study. Int J Eat Disord 2010, 43:35-41.

10. Keel PK: Purging disorder: Subthreshold variant or full-threshold eating disorder? Int J Eat Disord 2007, 40:S89-S94.

11. Keel PK, Striegel-Moore RH: The validity and clinical utility of purging disorder. Int J Eat Disord 2009, 42:706-719.

12. Heatherton TF, Mahamedi F, Striepe M, Field AE, Keel P: A 10-year longitudinal study of body weight, dieting, and eating disorder symptoms. J Abnorm Psychol 1997, 106:117-125.

13. Kotler LA, Cohen P, Davies M, Pine DS, Walsh BT: Longitudinal relationships between childhood, adolescent, and adult eating disorders J Am Acad Child Adolesc Psychiatry 2001, 40:1434-1440.

14. Patton GC, Coffey C, Sawyer SM: The outcome of adolescent eating disorders: Findings from the Victorian Adolescent Health Cohort Study. Eur Child Adolesc Psychiatry 2003, 12(Suppl 1):125-129.

15. Striegel-Moore RH, Rosselli F, Perrin N, DeBar L, Wilson GT, May A, et al: Gender difference in the prevalence of eating disorder symptoms. Int J Eat Disord 2009, 42:471-474

16. Anderson $C B$, Bulik CM: Gender differences in compensatory behaviors, weight and shape salience, and drive for thinness. Eat Behav 2004, 5:1-11.

17. von Soest T, Wichstrom L: Cohabitation reduces bulimic symptoms in young women. Int J Eat Disord 2006, 39:484-491.

18. Wichstrom L: Psychological and behavioral factors unpredictive of disordered eating: A prospective study of the general adolescent population in Norway. Int J Eat Disord 2000, 28:33-42.

19. Henderson $M$, Freeman CP: A self-rating scale for bulimia. The 'BITE'. Br J Psychiatry 1987, 150:18-24.

20. Garner DM, Olmsted MP, Bohr Y, Garfinkel PE: The eating attitudes test: psychometric features and clinical correlates. Psychol Med 1982, 12:871-878.

21. Lavik NJ, Clausen SE, Pedersen W: Eating behaviour, drug use, psychopathology and parental bonding in adolescents in Norway. Acta Psychiatr Scand 1991, 84:387-390.

22. Brown TA, Cash TF, Lewis RJ: Body-image disturbances in adolescent female binge purgers - a brief report of the results of a national survey in the USA. J Child Psychol Psychiatry Allied Discip 1989, 30:605-613.

23. Kandel DB, Davies M: Epidemiology of depressive mood in adolescents an empirical study. Arch Gen Psychiatry 1982, 39:1205-1212

24. Derogatis LR, Lipman RS, Rickels K, Uhlenhuth EH, Covi L: The Hopkins Symptom Checklist (HSCL): A self-report symptom inventory. Behav Sci 1974, 19:1-15.

25. Pedersen $W$, von Soest $T$ : Smoking, nicotine dependence and mental health among young adults: A 13-year population-based longitudinal study. Addiction 2009, 104:129-137.

26. Wichstrom L: Harters self-pereception profile for adolescents - reliability, validity, and evaluation of the question format. J Pers Assess 1995 65:100-116.
27. Harter S: Manual for the self-perception profile for adolescents Denver, CO: University of Denver; 1988

28. Parker $\mathrm{G}$, Tupling $\mathrm{H}$, Brown LB: A parental bonding instrument. $\mathrm{Br} J \mathrm{Med}$ Psychol 1979, 52:1-10.

29. Rosenberg M: Self-concept from middle childhood through adolescence. In Psychological perspectives on the self. Volume 3. Edited by: Suls D, Greenwald AG. Hillsdale, NJ: Lawrence Erlbaum; 1986:107-136.

30. Alsaker FD, Olweus D: Assessment of global negative self-evaluations and perceived stability of self in Norwegian preadolescents and adolescents. J Early Adolesc 1986, 6:269-278.

31. Sarason IG, Levine HM, Basham RB, Sarason BR: Assessing social support The social support questionnaire. J Pers Soc Psychol 1983, 44:127-139.

32. Russell D, Peplau LA, Cutrona CE: The revised UCLA loneliness scale: Concurrent and discriminant validity evidence. J Pers Soc Psychol 1980 39:472-480.

33. Goodman E, Hinden BR, Khandelwal S: Accuracy of teen and parental reports of obesity and body mass index. Pediatrics 2000, 106:52-58.

34. Austin SB, Ziyadeh NJ, Vohra S, Forman S, Gordon CM, Prokop LA, et al: Irregular menses linked to vomiting in a nonclinical sample: Findings from the national eating disorders screening program in high schools. J Adolesc Health 2008, 42:450-457.

35. Hay P, Fairburn C: The validity of the DSM-IV scheme for classifying bulimic eating disorders. Int J Eat Disord 1998, 23:7-15.

36. Nunez-Navarro A, Jimenez-Murcia S, Alvarez-Moya E, Villarejo C, Diaz IS, Augmantell CM, et al: Differentiating purging and nonpurging bulimia nervosa and binge eating disorder. Int J Eat Disord 2011, 44:488-496.

37. Lewinsohn PM, Seeley JR, Moerk KC, Striegel-Moore RH: Gender differences in eating disorder symptoms in young adults. Int J Eat Disord 2002, 32:426-440.

38. De Young KP, Lavender JM, Anderson DA: Binge eating is not associated with elevated eating, weight, or shape concerns in the absence of the desire to lose weight in men. Int J Eat Disord 2010, 43:732-736.

39. Keel PK, Baxter MG, Heatherton TF, Joiner TE: A 20-year longitudinal study of body weight, dieting, and eating disorder symptoms. J Abnorm Psychol 2007, 116:422-432.

40. Striegel-Moore RH, Franko DL: Epidemiology of binge eating disorder. Int J Eat Disord 2003, 34(Suppl):S19-S29.

41. Leon GR, Carroll K, Chernyk B, Finn S: Binge eating and associated habit patterns within college-student and identified bulimic populations. Int J Eat Disord 1985, 4:43-57.

42. Sierra-Baigrie S, Lemos-Giraldez S, Fonseca-Pedrero E: Binge eating in adolescents: its relation to behavioural problems and family-meal patterns. Eat Behav 2009, 10:22-28.

43. Lavender JM, De Young KP, Anderson DA: Eating Disorder Examination Questionnaire (EDE-Q): Norms for undergraduate men. Eat Behav 2010, 11:119-121.

44. Luce $\mathrm{KH}$, Crowther $\mathrm{JH}$, Pole M: Eating Disorder Examination Questionnaire (EDE-Q): Norms for undergraduate women. Int J Eat Disord 2008, 41:273-276.

45. Stice E: Risk and maintenance factors for eating pathology: A metaanalytic review. Psychol Bull 2002, 128:825-848.

46. Stice E, Killen JD, Hayward C, Taylor CB: Support for the continuity hypothesis of bulimic pathology. J Consult Clin Psychol 1998, 66:784-790.

47. Crowther JH, Armey M, Luce KH, Dalton GR, Leahey T: The point prevalence of bulimic disorders from 1990 to 2004. Int J Eat Disord 2008, 41:491-497.

48. Norval DG: Distinguishing Age, Period, and Cohort Effects. In Handbook of the Life Course. Edited by: Jeylan TM, Michael JS. New York: Kluwer Academic; 2003:465-476.

\section{Pre-publication history}

The pre-publication history for this paper can be accessed here: http://www.biomedcentral.com/1471-2458/12/32/prepub

doi:10.1186/1471-2458-12-32

Cite this article as: Abebe et al:: Binge eating, purging and non-purging compensatory behaviours decrease from adolescence to adulthood: A population-based, longitudinal study. BMC Public Health 2012 12:32. 